\title{
Effect of water content in solvent on the critical temperature in the formation of self-assembled hexadecyltrichlorosilane monolayers on mica
}

\author{
Li-Jen Chen *, Yi-Hsing Tsai, Chuan-Sheng Liu, \\ Dah-Renn Chiou, Ming-Chih Yeh \\ Department of Chemical Engineering, National Taiwan University, Taipei 106, Taiwan
}

Received 20 December 2000; in final form 20 August 2001

\begin{abstract}
It is found that the critical temperature in the formation of self-assembled $n$-hexadecyltrichlorosilane (HTS) monolayers on mica depends on the water content in the solvent dodecane. Under the condition of $35 \pm 2 \mathrm{ppm}$ water content in dodecane, the critical temperature is found at $31 \pm 1{ }^{\circ} \mathrm{C}$. When the water content in dodecane is lowered down to $13 \pm 2 \mathrm{ppm}$, no critical temperature is found in the temperature range of $10-55^{\circ} \mathrm{C}$. Our results indicate that the critical temperature is subject to the extent of hydrolysis of HTS molecules in bulk phase of solution. A reaction mechanism of monolayer formation is discussed. (c) 2001 Elsevier Science B.V. All rights reserved.
\end{abstract}

\section{Introduction}

Self-assembled monolayers (SAMs) have potential applications in several fields, such as biosensors, wetting, adhesion, lubrication, chromatography, and lithography [1-5]. A specific system, self-assembled alkyltrichlorosiloxane monolayers on hydroxylated surfaces, has been the subject of intense investigations since their discovery by Sagiv [6] in 1980. The mechanism of the growth kinetics of selfassembled alkyltrichlorosiloxane monolayers remains controversial. Note that there are so many system parameters, such as temperature, water content in the adsorbate solution, adsorbate concentration in the adsorbate solution, substrate,

\footnotetext{
${ }^{*}$ Corresponding author. Fax: +886-2-23623040.

E-mail address: ljchen@ccms.ntu.edu.tw (L.-J. Chen).
}

pretreatment of substrate, water content at the substrate surface, etc., far from completely under control [7-21].

The amount of water in the solution and at the substrate surface has a strong effect on the packing and conformation of monolayers $[7,13,17,19]$. The self-assembly process requires a certain amount of water at the substrate surface to hydrolyze the alkyltrichlorosilane molecules. It was demonstrated that self-assembled octadecyltrichlorosilane (OTS) monolayers do not form complete monolayer on dehydrated silica [8,9] and on dehydrated mica [10]. In addition, the water layer at the substrate surface acts as a lubricating layer to allow adsorbed molecules to laterally diffuse and aggregate by Brownian motion [11]. Angst and Simmons [12] pointed out that hydrated silica surfaces produce close-packed OTS monolayers with a critical surface tension of $20.3 \mathrm{mN} / \mathrm{m}$ while 
monolayer coverage diminishes with a critical surface tension of $24.3 \mathrm{mN} / \mathrm{m}$ for dry surfaces. According to the advancing contact angle of dodecane on a silanated wafer, it was found [7] that water in solution is necessary in a small quantity to form a complete monolayer. Lambert et al. [19] also pointed out that the use of highpurity anhydrous solvent is one of the essential prerequisities for reproducible OTS monolayers. Vallant et al. [13] reported that an increase of water content in solution would accelerate the growth rate of self-assembled OTS monolayers.

Besides water content, the reaction temperature is also essential in the formation of SAMs [7]. It was found that there exists a critical temperature, $T_{\mathrm{c}}$, in the formation of self-assembled alkylsiloxane monolayers on silica [14]. That is, when the monolayer is prepared below $T_{\mathrm{c}}$, the structure of monolayers is more highly packed and ordered with a critical surface tension of $20 \pm 0.5 \mathrm{mN} / \mathrm{m}$. In contrast, when the monolayer is prepared above $T_{\mathrm{c}}$, the structure of monolayer becomes conformational disorder. In addition, the critical surface tension of the monolayer increases with increasing preparation temperature. According to Brzoska et al. $[14,15]$ the critical temperature is analogous to the triple point in the phase diagram of Langmuir monolayers. On the other hand, Rye [16] pointed out that the melting points of the corresponding linear alkanes are in much better agreement with the experimental critical temperatures.

Based on either the viewpoint of triple point $[11,14,15]$ or that of melting point [16], the critical temperature is an intrinsic property of the alkyltrichlorosilane molecules and consequently irrelevant to the substrate and the solvent used for the reaction. In this study, we therefore changed the substrate from silica to mica and tried to explore whether the critical temperature in the formation of self-assembled hexadecyltrichlorosilane (HTS) monolayers on mica is the same as that on silica. In addition, the effect of water content on the formation of HTS monolayers was carefully explored. It is interesting to find out that the critical temperature in the formation of self-assembled HTS monolayers on mica is different from that on silica and varies as a function of the water content in the solvent dodecane.

\section{Experimental section}

Dodecane (Merck, >99\%) was purified by percolating through a column of anhydrous aluminum oxide (Merck). This percolating process also removed certain amount of water in dodecane. The water content of dodecane was then determined by Karl-Fischer titration (MKC-210, Kyoto Electronics, Japan) and found to be $13 \pm 2 \mathrm{ppm}$. Dodecane was then used as a solvent to prepare the hexadecyltrichlorosilane (United Chemical Technologies) solution. Dodecane was heated or cooled to reach a prescribed temperature, and then the HTS solution was freshly prepared right before the silanation reaction. During the silanation reaction, the HTS solution was kept in a double-walled Pyrex vessel thermostated at the prescribed temperature by circulating water. The water circulating through the vessel jacket was completely isolated from the HTS solution and did not introduce any water to the HTS solution. Both sides of $1 \times 3 \mathrm{~cm}$ muscovite mica slide (Agar Scientific, UK) were freshly cleaved, then exposed to steam for around $30 \mathrm{~s}$ until water drops formed on the surface, and then dried with nitrogen gas. The hydrated mica slides were immersed in a $1 \mathrm{mM}$ solution of HTS for at least one hour and then removed and rinsed by dichloromethane (Merck, 99.5\%) and trichloromethane (Merck, 99-99.4\%) to remove any un-adsorbed HTS molecules. The water content of the HTS solutions after the silanation reaction was found to be increased by $4 \pm 1 \mathrm{ppm}$. The reaction temperature was in the range of $10-55{ }^{\circ} \mathrm{C}$. All the preparations of the adsorbate (HTS) solution and silanation reactions were conducted in a glove-bag filled with dry nitrogen to exclude the amount of water traces in the surrounding atmosphere. Structural feature of monolayers was characterized by advancing contact angle and AFM measurements.

A homemade enhanced video-microscopy system incorporated with a digital image analysis technique was applied to measure advancing contact angles. The details of the methodology and its experimental setup can be found elsewhere [22-24]. The accuracy on the advancing contact angle measurements is better than $\pm 0.1^{\circ}$, even for small angles. 
AFM measurements were performed in air with a NanoScope IIIa AFM (Digital Instruments, Santa Babara, USA). Commercial silicon cantilevers (Nanosensors, Germany) with typical spring constants of $21-78 \mathrm{~N} / \mathrm{m}$ were used to operate the AFM in tapping mode.

\section{Results and discussion}

In order to explore the effect of water content on the formation of HTS monolayers, two different water contents in dodecane were used to prepare the HTS monolayers on mica. Fig. 1 shows the results of the critical surface tension of the HTS monolayer as a function of the reaction temperature at two different water contents, $13 \pm 2$ and $35 \pm 2 \mathrm{ppm}$. It is interesting to find out that the critical temperature in the formation of selfassembled HTS monolayers on mica depends on the water content in dodecane.

Under the condition of $35 \mathrm{ppm}$ water content in dodecane, the critical temperature is found at $31.0 \pm 1.0{ }^{\circ} \mathrm{C}$, which is higher than that of HTS on silica, $24.0 \pm 1.5^{\circ} \mathrm{C}[1,2]$. When the HTS mono-

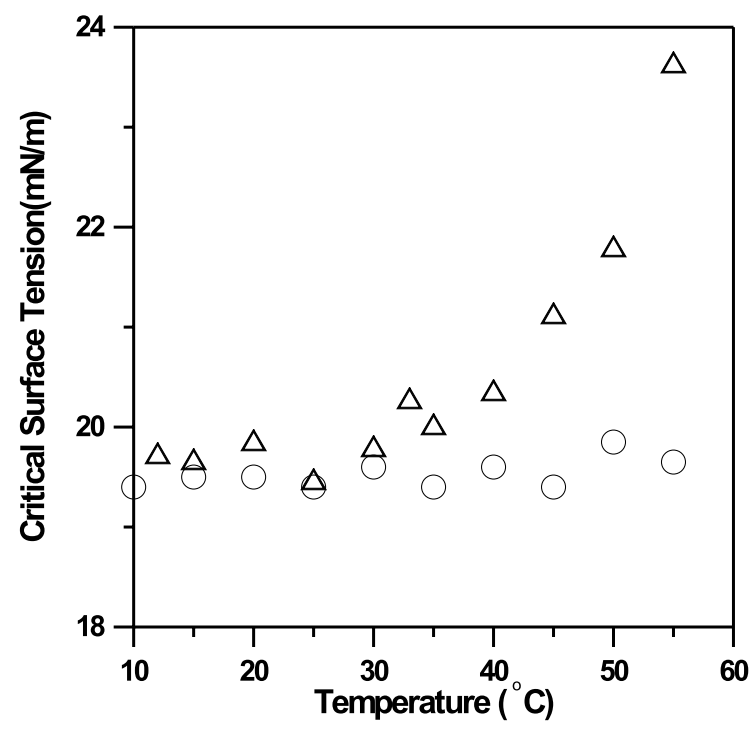

Fig. 1. Variation of the critical surface tension of self-assembled HTS monolayers on mica as a function of the preparation temperature at two different water contents in dodecane, 35 $\operatorname{ppm}(\triangle)$ and $13 \mathrm{ppm}(\bigcirc)$. layer is prepared below $T_{\mathrm{c}}\left(31 \pm 1^{\circ} \mathrm{C}\right)$, the structure of monolayers is more highly packed and ordered with a critical surface tension of $19.7 \pm 0.2$ $\mathrm{mN} / \mathrm{m}$. On the other hand, when the monolayer is prepared above $T_{\mathrm{c}}$, the structure of monolayer becomes conformational disorder and the critical surface tension of the monolayer increases with increasing preparation temperature.

When the water content in dodecane is lowered down to $13 \mathrm{ppm}$, the structure of monolayers is always highly packed and ordered with a critical surface tension of $19.7 \pm 0.3 \mathrm{mN} / \mathrm{m}$ over the whole experimental temperature range, as shown in Fig. 1. That is, no critical temperature is found in the temperature range of $10-55^{\circ} \mathrm{C}$ for the water content of $13 \mathrm{ppm}$.

Water is necessary for hydrolysis of HTS molecules, which can occur either in the bulk phase of solution or at the substrate surface. Sagiv [6] suggested that if the only water present in the system is at the substrate surface, then the OTS can only hydrolyze at the surface in favor of the formation of well-defined SAMs. In this study, the only difference between two experimental sets is the water content in the solvent dodecane. Our results indicate that the critical temperature is subject to the extent of hydrolysis of HTS molecules in the bulk phase of solution.

In fact, the water contents of 35 and of $13 \mathrm{ppm}$ are equivalent to, respectively, 1.5 and $0.54 \mathrm{mM}$ water in dodecane. Consider the $1 \mathrm{mM}$ HTS solution. Every two HTS molecules can be hydrolyzed by three water molecules in the bulk phase of solution for the water content of $35 \mathrm{ppm}$. On the other hand, almost every two HTS molecules can be hydrolyzed by only one water molecule in the bulk phase of solution for the water content of $13 \mathrm{ppm}$. As a consequence, the polymerization of hydrolyzed species in the bulk phase of solution undergoes to a greater extent for the water content of $35 \mathrm{ppm}$ than that for the water content of $13 \mathrm{ppm}$. Once the clusters formed by a three-dimensional polymerization of hydrolyzed species in the bulk phase of solution deposit on the substrate surface, that prevents the formation of well-defined SAMs. Vallant et al. [13] also found out that a decrease of the water content in the adsorbate solution would reduce the deposition of the OTS clusters. 
The AFM images of HTS films on mica prepared at $35^{\circ} \mathrm{C}$ from solutions with different water contents, 11 and 37 ppm, are shown in Figs. 2a and $b$, respectively. The dimension of the images is

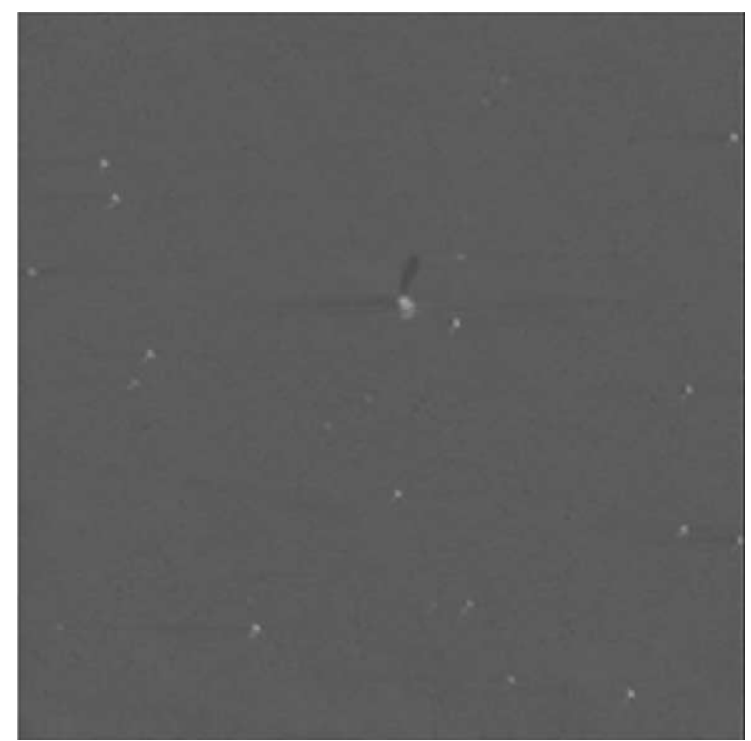

(a)

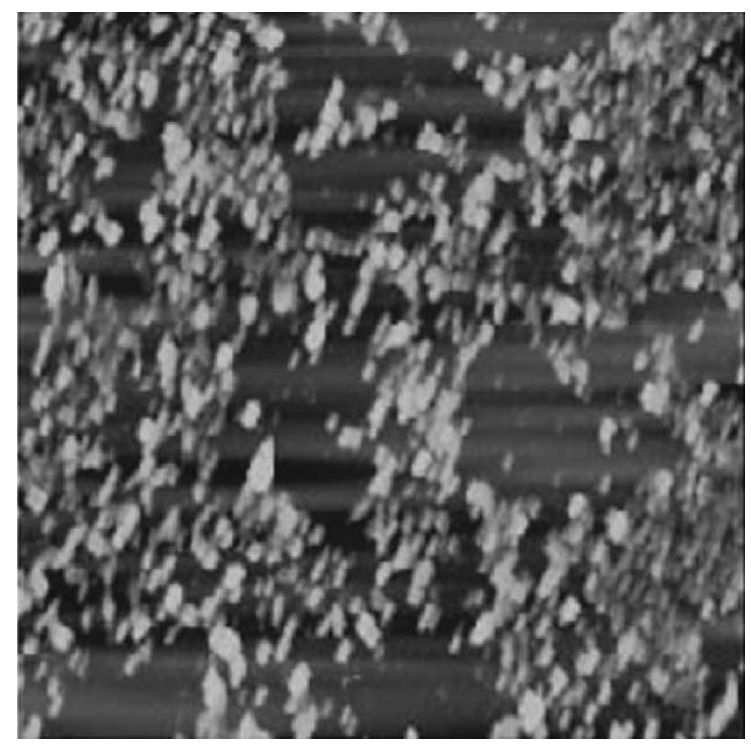

(b)

Fig. 2. AFM images $(10 \times 10 \mu \mathrm{m})$ of HTS films on mica prepared at $35^{\circ} \mathrm{C}$ by immersing for $1 \mathrm{~h}$ in freshly prepared HTS solutions with different water contents in dodecane: (a) $11 \mathrm{ppm}$ and (b) 37 ppm.
$10 \times 10 \mu \mathrm{m}$. As expected, for the low water content, a smooth flat surface was obtained, as illustrated in Fig. 2a. On the other hand, it is obvious that there are lot of clusters deposited on the surface for the high water content, as illustrated in Fig. 2b, consistent with the results of Xiao et al. [18] for the octadecyltriethoxysilane SAM formation on mica.

According to the Arrhenius law, the reaction rate increases along with the temperature. Therefore, when the silanation temperature is increased, both the number and the size of clusters formed by the polymerization of hydrolyzed species in the bulk phase of solution are enhanced. The probability of deposition of these clusters on the mica surface is also increased along with the temperature. The deposition of these clusters on the mica surface leads to an incomplete monolayer. The critical temperature happens to be the threshold of temperature of cluster deposition. Beyond this threshold of temperature, the cluster deposition is so serious that no well-defined SAM is formed. Below this threshold of temperature, the reaction rate of hydrolysis and polymerization in the bulk phase of solution is slow enough and the effect of cluster deposition on the SAM formation is negligible. Therefore, the well-defined SAM is always obtained at low temperatures.

At low water contents in dodecane, the reaction rate of cluster formation by a three-dimensional polymerization of hydrolyzed HTS molecules in the bulk phase of solution is slow enough. Consequently, there is ample time for the HTS molecules (or the hydrolyzed HTS molecules) to diffuse onto the mica surface to hydrolyze, to graft, and to form a monolayer onto the mica surface without the competition of the cluster deposition. Our results of the low water content (13 ppm) demonstrate that highly packed and ordered SAM is obtained even at high temperatures, as high as $55{ }^{\circ} \mathrm{C}$. It is plausible to conjecture that there maybe exists a critical temperature for the system of the water content of $13 \mathrm{ppm}$ and this critical temperature occurs at whenever the effect of the cluster deposition becomes pronounced and considerable, must be somewhere higher than $55^{\circ} \mathrm{C}$.

In conclusion, the effect of water content in the solvent dodecane on the critical temperature in the 
formation of self-assembled HTS monolayers on mica is explored. It is found that the critical temperature depends on the water content in dodecane. The higher the water content in dodecane, the lower the critical temperature. The origin of existence of the critical temperature is due to the deposition of clusters formed by a three-dimensional polymerization of hydrolyzed HTS molecules in the bulk phase of solution on the mica surface.

\section{Acknowledgements}

This work was supported by the National Science Council of Taiwan, Republic of China.

\section{References}

[1] A. Ulman, An Introduction to Ultrathin Organic Films, Academic Press, Boston, 1991.

[2] L.H. Dubois, R.G. Nuzzo, Annu. Rev. Phys. Chem. 43 (1992) 437.

[3] J. Andle, J. Vetelino, R. Lec, D. McAllister, in: Proc. IEEE Ultrasonics Symp., 1989, p. 579.

[4] J.D. Swalen, D.L. Allara, J.D. Andrade, E.A. Chandross, S. Garoff, J. Israelachvili, T.J. McCarthy, R. Murray, R.F. Pease, J.F. Rabolt, K.J. Wynne, H. Yu, Langmuir 3 (1987) 932.
[5] Y. Xia, G.M. Whitesides, Angew. Chem. Int. Ed. 37 (1998) 550.

[6] J. Sagiv, J. Am. Chem. Soc. 102 (1980) 92.

[7] P. Silberzan, L. Leger, D. Ausserre, J.J. Benattar, Langmuir 7 (7) (1991) 1647.

[8] C.P. Tripp, M.L. Hair, Langmuir 8 (1992) 1120.

[9] C.P. Tripp, M.L. Hair, Langmuir 11 (1995) 149.

[10] G. Carson, S.J. Granick, Mater. Res. 5 (1990) 1745.

[11] A.N. Parikh, D.L. Allara, I.B. Azouz, F. Rondelez, J. Phys. Chem. 98 (1994) 7577.

[12] D.L. Angst, G.W. Simmons, Langmuir 7 (1991) 2236.

[13] T. Vallant, H. Brunner, U. Mayer, H. Hoffmann, T. Leitner, R. Resch, G. Friedbacher, J. Phys. Chem. B 102 (1998) 7190.

[14] J.B. Brzoska, N. Shahidzadeh, F. Rondelez, Nature 360 (1992) 719.

[15] J.B. Brzoska, I. Ben Azouz, F. Rondelez, J. Phys. Chem. 90 (1994) 3054.

[16] R.R. Rye, Langmuir 13 (1997) 2588.

[17] J. Peanasky, H.M. Schneider, S. Granick, C.R. Kessel, Langmuir 11 (1995) 953.

[18] X.-D. Xiao, G.-Y. Liu, D.H. Charych, M. Salmeron, Langmuir 11 (1995) 1600.

[19] A.G. Lambert, D.J. Neivandt, R.A. McAloney, P.B. Davies, Langmuir 16 (2000) 8377.

[20] C.R. Kessel, S. Granick, Langmuir 7 (1991) 532.

[21] T. Nakagawa, K. Ogawa, T. Kurumizawa, Langmuir 10 (1994) 525.

[22] A. Amirfazli, D.Y. Kwok, J. Gaydos, A.W. Neumann, J. Colloid Interface Sci. 205 (1998) 1.

[23] M.-C. Yeh, L.-J. Chen, S.-Y. Lin, C.-T. Hsu, J. Chin. Inst. Chem. Eng. 32 (2001) 109.

[24] S.-Y. Lin, L.-J. Chen, J.-W. Xyu, W.-J. Wang, Langmuir 11 (1995) 4159. 